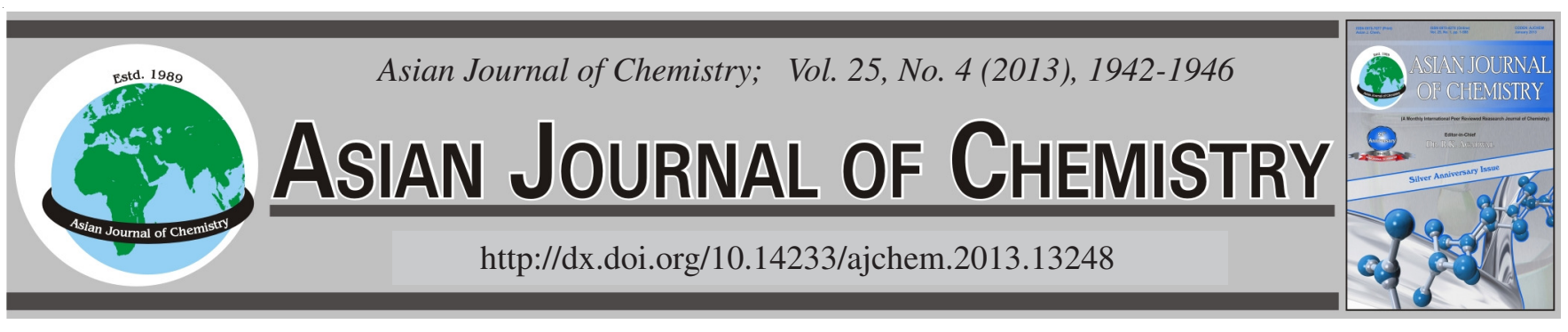

\title{
Determination of Benzoyl Peroxide in Flour Brightener with Microfluidic Chip-Based Flow-Injection Chemiluminescence
}

\author{
Xiaozhang Wang, Chaohui Wang ${ }^{*}$, Qunming Zhang, Jing Wang and Tengfei Zheng
}

School of Mechanical Engineering Xian Jiaotong University, Xi'an 710049, P.R. China

*Corresponding author: Tel: +86 29 82668616; E-mail: chhw@mail.xjtu.edu.cn

(Received: 14 December 2011;

Accepted: 8 October 2012)

AJC-12255

\begin{abstract}
Benzoyl peroxide (BPO) in flour brightener was determined with chip-based chemiluminescence reaction. The microfluidic chip was fabricated with PDMS using MEMS techniques and the flow-injection chemiluminescence experiments were performed. The conditions of experiments including sample inject and flow-rate were selected and optimized according to their different influences on detect results. The standard curve of relative chemiluminescence intensity and benzoyl peroxide concentration was constructed from the reaction of benzoyl peroxide and luminol solutions with specific concentration, respectively. The experimental results indicate that a good linear relation is formed between the chemiluminescence intensity and benzoyl peroxide concentration within $10^{-6}-10^{-8} \mathrm{~g} \mathrm{~mL}^{-1}$. The detection limit is about $2 \times 10^{-9} \mathrm{~g} \mathrm{~mL}^{-1}$ in benzoyl peroxide solution. At last, flour brightener samples bought from market were tested with this microfluidic chip system. The result was consistent well with the content of brightener tag. Some important topics of integrated chip were discussed including inject pump, active mixer and microheater.
\end{abstract}

Key Words: Microfluidic chip, Chemiluminescence, Flow injecting, Benzoyl peroxide.

\section{INTRODUCTION}

Benzoyl peroxide (BPO) is the most widely used flour brightener in the flour industry since the last century because it can improve the quality of the raw flour with only a little damage of nutrition. But the biological and medical research show that the overdose and abuse of benzoyl peroxide will harm the health of people who eat the flour regularly. It suggests that assimilating large quantity benzoyl peroxide in long time will do harm to liver and lead to many diseases, while in short time will make people in toxic symptom including feeling sick, dizzy and neurasthenic etc. ${ }^{1,2}$. Although benzoyl peroxide draws the interest of researchers for decades, the absence of the related exterminating criterion and method make it a difficult mission to prevent abuse. The main methods of detecting benzoyl peroxide are liquid chromatography, capillary electrophoresis, colorimatry, iodometry and ultralviolet spectrophotometry ${ }^{3,4}$. These methods can produce good results by utilizing expensive equipments and laboratory conditions, which in turn limit their applications because of without in situ detecting, real time analysis and quick determination.

Chemiluminescence (CL) can be used in micro-quantity substances analysis utilizing their unique luminescence property in intensity or spectrum. Without the outside inter- ference, chemiluminescence detect even can do photon metrology and achieve high detect limit $\left(10^{-12}-10^{-21} \mathrm{~mol}\right)$ and wide linearity (3-6 numerical scale) $)^{5-7}$. Owing to these advantages chemiluminescence is widely applied and quickly developed in environment monitoring, pesticide detecting, pharmacy selecting and drug abuse ${ }^{7-10}$. Microfluidic chip is a novelty equipment in many applications with the advantages of high output, small dead volume, less reagent consuming, low cost and easy use ${ }^{11-13}$. Zhang et al..$^{13,14}$ investigated the determination of nitrite, benzoyl peroxide and cyanide with their home-made microfluidic chip and demonstrated the application of chemiluminescence detect. The Integrated chemiluminescence chip also draws researchers interest. Jorgensen et al. ${ }^{15}$ integrated a back-side photodiodes with a microfluidic channel network on a silicon substrate and tested in flow-injection chemiluminescence detection successfully. Wang et al. ${ }^{16}$ demonstrated that thin-film organic photodiodes can be used as integrated detector for antioxidant on-chip chemiluminescence detection. Guan et $\mathrm{al} .{ }^{17}$ used capillary and evaporation effects as micropump and integrated in a microfluidic flow injection chemiluminescence system and an good average flow-rate was achieved in luminol reaction. Zhao et al..$^{18}$ developed a microchip using electrophoresis and chemiluminescence detection together to determine carnosinerelated peptides with a micromole level quantification. 
In this paper, a chip-based flow injection chemiluminescence analysis system was employed to determinate the concentration of benzoyl peroxide in flour brightener. The microchip was made of transparent PDMS using micro-molding and sealed with plasma oxidization. Flow injection chemiluminescence experiments were carried out to investigate the different effects of flow conditions. The influence of fluid injection and flow-rate were observed to get a appropriate condition for injection. This research aims at an optimized flow injection chemiluminescence conditions for microchip based substance detecting. Combining the high sensitivity and selectivity of chemiluminescence, high output, less consumption and low cost of micro-chip, a quick real time determination will be achieved. The integrated detect chip with sample injecting, flow controlling, active mixing, reaction regulation and other related parts is the most expected device for chemiluminescence detection. Some related points about the integrated chemiluminescence chip were also discussed such as solution injector, active mixer and microheater. The advantages of the potential candidates are also presented.

\section{EXPERIMENTAL}

The fluid and reagents were pumped into microchip by IFFM-D fluid injector (Xian Remax Analysis Instrument Co. Ltd. China) and the chemiluminescence intensity was measured by a IFFS-A microfluidic flow chemiluminscence analyzer (Xian Remax Analysis Instrument Co. Ltd. China), which can detect the chemiluminescence intensity in a spectrum from $300-650 \mathrm{~nm}$. The results were recorded and related parameters of chemiluminescence are calculated by a selfrelied software of the instrument. Syringe pump ALC-IP800 (Shanghai Alcott Biotech. Co. Ltd. China) was also used as injecor for comparison.

Benzoyl peroxide was bought from Xi'an Chemical Reagent Factory and luminol was bought from Acorus. The benzoyl peroxide standard solution was prepared by dissolving $0.05 \mathrm{~g}$ dry benzoyl peroxide in $50 \mathrm{~mL}$ absolute ethyl alcohol to get a $0.001 \mathrm{~g} \mathrm{~mL}^{-1}$ benzoyl peroxide standard. Then the series of solutions of different concentration were diluted sequentially with the ethyl alcohol. The $0.01 \mathrm{~mol} \mathrm{~L}^{-1}$ luminol solution was prepared also by dissolving $1.772 \mathrm{~g}$ lumiol in $1 \mathrm{~L} 0.1 \mathrm{~mol} \mathrm{~L}^{-1} \mathrm{NaOH}$ solution and stocked for use. All the reagents were of analytical reagent grade and home-made bidistilled water was used for dilution.

Fabrication of the microchip: Microchip was fabricated with micromolding using SU-8 photo resistor (Microchem Co. USA) and PDMS Sylgard184 (Dow Coring Co. USA). The sequences include spinning, pre-baking, photolithographing, post-baking, developing, molding, curing and bonding. The SU-8 mold was etched and developed on a silicon wafer (400 $\mu \mathrm{m} \times \varnothing 100 \mathrm{~mm}$, Mchemiluminescence Electronic Material. Luoyang China). Then the special mixture of PDMS and curing solution were perfused on the fabricated mold. The microstructure of the chip was cured in a hot box with a special temperature and minutes according to the instructions. When the curing was over, PDMS structure was lifted off from SU-8 mold and assemble together to seal the channel. Fig. 1 shows the completed chip with width and length $40 \mathrm{~mm} \times 60$ $\mathrm{mm}$, which has two inlets and one outlet for two kinds of fluid mixing and chemiluminescence detection. The inset image is a microscopic figure of channel in microchip. The convexes on the both side of sidewalls can squeeze and twist the streamlines of fluids to promote mixing and chemiluminescence reaction. The microchannel in chip is rectangular with width $500 \mu \mathrm{m}$ and height $40 \mu \mathrm{m}$, respectively. The convex is $600 \mu \mathrm{m}$ both in length and width with an interval of $2.5 \mathrm{~mm}$.

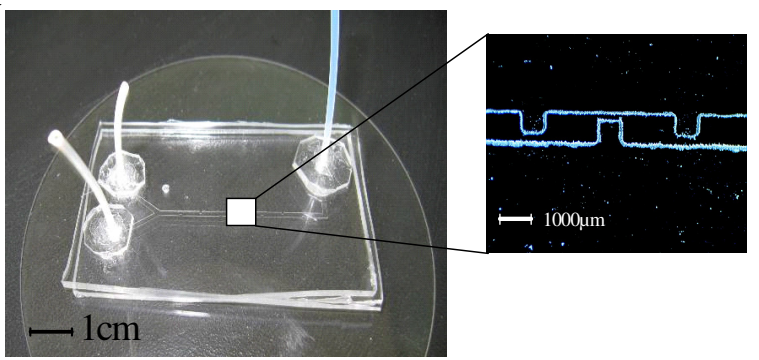

Fig. 1. Fabricated microfuidic chip. Inset image is a microscopic image of channel in chemiluminescence chip

Four pieces of flour brightener sample were bought from market randomly. Brighteners were dried and dissolved in absolute ethyl alcohol to make sample solution. For instance, $1 \mathrm{~g}$ brightener was put into $100 \mathrm{~mL}$ alcohol and agitated for thoroughly dissolving and centrifugal separation was utilized to get the upper solution for use.

Procedure and operation: The microchip was flushed with acetone, ethyl alcohol and distilled water was used subsequently to remove the contamination and dust in channel. Then the chip was put on the detecting window of analyzer and the inlet and outlet pipes were connected tightly to prevent leaking during experiments.

There were two kinds solutions to be injected into microchip, one is benzoyl peroxide and the other luminol solution. The former was perfused constantly and the latter was injected with a pulse mode. First, the main pump of IFFM-D was started. The benzoyl peroxide sample was pumped into the microchannel and the flow-rate became steady after several minters. As the flow-rate became smooth, the minor pump would be controlled to start and stop according to a preassigned scheme. The two pumps were controlled separately to achieve different sample volume injection. The chemiluminescence intensity was recorded by the self-relied software and post-process was performed to calculate the related results of chemiluminescence. The main pump also was replaced with the syringe pump for comparison.

\section{RESULTS AND DISCUSSION}

Luminol reacts with peroxide and emits photons in alkaline medium ${ }^{10-14}$. benzoyl peroxide-luminol chemiluminescence system emits near the wavelength of $425 \mathrm{~nm}^{19}$. The chemiluminescence intensity varies with the concentrations of reacting solution and the influence of fluid injection, flow-rate and others should not be neglected to optimize the detecting application.

Effect of fluid injection: The most useful method is pressure difference between inlet and outlet to drive fluid flowing through microchannel. Both the syringe and peristaltic pump 
can be used. In order to investigate the effects of two pumps, experiments were performed with standard benzoyl peroxide and luminol solutions, while concentration of the two reactors were $0.001 \mathrm{~g} \mathrm{~mL}^{-1}$ and $0.01 \mathrm{~mol} \mathrm{~L}^{-1}$, respectively. The recorded results were plotted in Fig 2. In this figure, the horizon axis indicates the number of experiments and the vertical axis describes the undimensional intensity of chemiluminescence, which is calculated by dividing the average value of 12 experiments. This figure shows that the peristaltic pump conducts less fluctuation of chemiluminescence intensity with a small relative deviation of $6 \%$. On the contrary, the syringe pump leads to a much larger fluctuation than its counterpart with a relative deviation of $30 \%$. This must be attributed to their different work mechanism. Syringe pump works continuously as the piston moves along the slider. Peristaltic pump works in a pulse-like mode although the spindle rotates consecutively. The former can not be controlled freely to start and stop injecting as the latter does. This result shows that peristaltic pump injection leads to a stable flow and chemiluminescence emission and can be applied in microchip chemiluminescence detection.

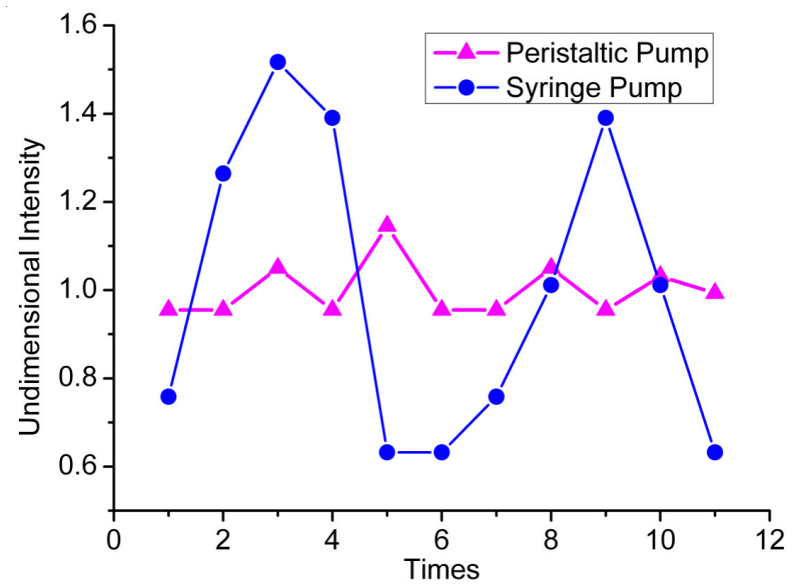

Fig. 2. Intensity of two ways sample injection: peristaltic pump and syringe pump

Injection rate selection: Due to the scale effect, microflow are much different from the macroflow. Therefore flow-rate of injection influences the chemiluminescence reaction evidently. Because of the constant channel length, if the flow-rate is high it flows through the channel quickly without chemical reaction and intensity emission. On the contrary, while flow-rate is low the chemiluminescence emission will be conducted with large reagents and time- consuming. In order to get a higher intensity in the detector, the flow-rate of minor pump in IFFM-D was adjusted and measured carefully. The results shows that controlling the angle velocity and operation time of peristaltic pump will obtain a steady chemiluminescence reaction and recognizable intensity. In this research, the minor peristaltic pump was set working on a angle velocity of $75 \mathrm{rpm}$, injecting $1 \mathrm{~s}$ and resting $20 \mathrm{~s}$. The recorded chemiluminescence intensity-time curve was showed in Fig. 3.

Chemiluminescence reproductiveness: The above conditions were applied and six replication experiments were recorded to demonstrate the reproductiveness of the microchip flow injection chemiluminescence. The observed results were

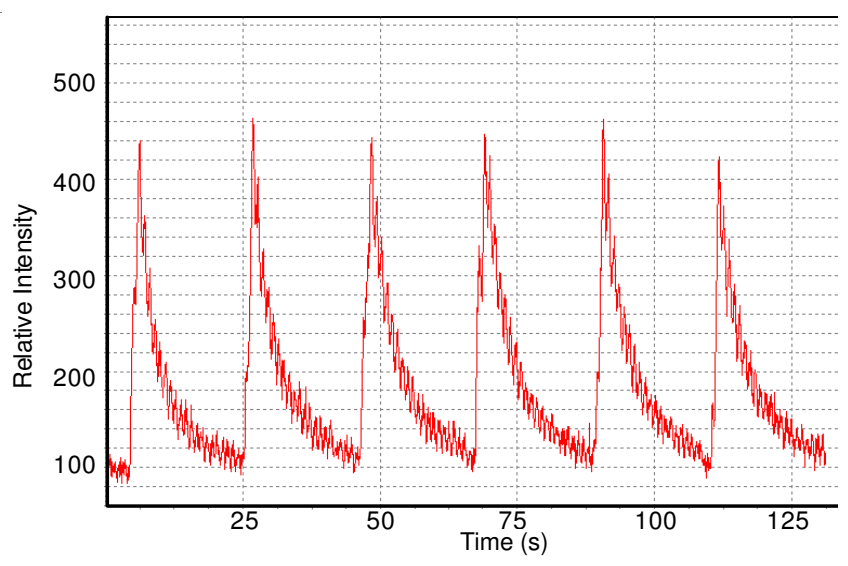

Fig. 3. Relative intensity for $1.0 \times 10^{-7} \mathrm{~g} \mathrm{~mL}^{-1}$ benzoyl peroxide and 0.001 mol L-1 luminol

shown in Fig. 3. with the concentration of benzoyl peroxide standard $1.0 \times 10^{-7} \mathrm{~g} \mathrm{~mL}^{-1}$ and lumiol $0.001 \mathrm{~mol} \mathrm{~L}^{-1}$. The room temperature was maintained at $c a .22^{\circ} \mathrm{C}$. The average recorded relative intensity of six experiments is 455.33 with a relative standard deviation of $2.9 \%$. This result is much better than the common requirement of detection.

Chemiluminescence curve and detection limit: In this research the chemiluminescence curve was determined by measuring $0.001 \mathrm{~mol} \mathrm{~L}^{-1}$ luminol standard and benzoyl peroxide solutions with various concentration. Experiments were carried out under the above conditions and the average intensity of each concentration sample was calculated. The measured results were processed and analyzed to obtain the inherent relation between concentration and intensity. It was found that the chemiluminescence intensity is linear over a wide range $10^{-6}-10^{-8} \mathrm{~g} \mathrm{~mL}^{-1}$ benzoyl peroxide concentration with the regression equation $\Delta \mathrm{I}=301.477 \times \mathrm{C}\left(10^{-6} \mathrm{~g} \mathrm{~mL}^{-1}\right)+351.221$ $(\mathrm{r}=0.99463, \mathrm{n}=7)$, where $\mathrm{C}$ is the concentration of benzoyl peroxide. The chemiluminescence curve of relative intensity by benzoyl peroxide concentration was plotted in Fig. 4. According to the IUPAC the benzoyl peroxide sample with $1 \times 10^{-7} \mathrm{~g} \mathrm{~mL}^{-1}$ was determined repeatedly and the detection limit was calculated as $2 \times 10^{-9} \mathrm{~g} \mathrm{~mL}^{-1}$.

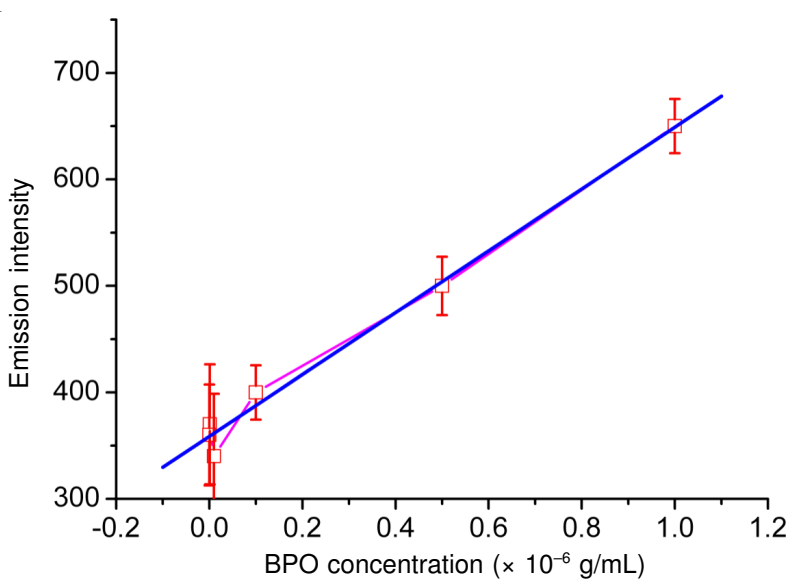

Fig. 4. Chemiluminescence intensity by the standard benzoyl peroxide concentration

Benzoyl peroxide in flour brightener: The prepared brightener solution was divided into three pieces and diluted 
TABLE-1

RESULTS OF FLOUR BRIGHTENER CHEMILUMINESCENCE DETECTION

\begin{tabular}{lccc}
\hline \multicolumn{1}{c}{ Concentration of brightener $\left(\mathrm{g} \mathrm{mL}^{-1}\right)$} & $1.0 \times 10^{-5}$ & $5.0 \times 10^{-6}$ & $1.0 \times 10^{-6}$ \\
\hline & 1900 & 1000 & 500 \\
& 2050 & 1030 & 540 \\
Relative chemiluminescence intensity & 1750 & 1070 & 550 \\
& 1890 & 1020 & 490 \\
& 1860 & 1040 & 520 \\
\hline Average intensity & 1890 & 1032 & 520 \\
Benzoyl peroxide concentration $\left(\mathrm{g} \mathrm{mL}^{-1}\right)$ & $5.1041 \times 10^{-6}$ & $2.2581 \times 10^{-6}$ & $5.5984 \times 10^{-7}$ \\
Content of benzoyl peroxide in brightener & $51.04 \%$ & $45.16 \%$ & $55.98 \%$ \\
\hline
\end{tabular}

with absolute ethyl alcohol to concentration of $1.0 \times 10^{-5}, 5.0$ $\times 10^{-6}$ and $1.0 \times 10^{-6} \mathrm{~g} \mathrm{~mL}^{-1}$, respectively, for chemiluminescence determination. Experiments were performed under the above conditions. The detection results were listed in Table-1. From this results the determined benzoyl peroxide content in flour brightener is $45-55 \%$, which is a little higher than the tag lable $30-40 \%$. This result demonstrates the accuracy of microfluidic chip flow injection chemiluminescence in detremination the benzoyl peroxide content of flour.

Discussion of integrated chemiluminescence chip: Chemiluminescence detect has many advantages in chemical and biological applications. Microfluidic chip makes these applications more convenient and low cost in works and researches. However, there remain some problems unsettled such as sample injection, mixing and reaction regulation.

Injection and flow-rate: Sample preparation and injection are important in detect and analysis. Hitherto, the reported papers show that all the chemiluminescence detect chips depend on the outer pump, syringe or peristalsis, to inject solution and conduct reaction. But, this microfluidic system is inconvenient for use because of the complex pipe connection and pump operation. As a result, this also restricts the applications of chemiluminescence detect in some conditions. Therefore, integrated pump in chip is much more preferred, which should inject sample with adjustable flow-rate to fulfill different requirements. In present experiments, the peristaltic pump injection shows much better performance than syringe pump. So the integrated pump in microchip must have the similar work scheme as the peristaltic pump. The piezoelectricactuated micro pump seems to be a good choice due to their similar work characteristics. The electroosmotic flow seems to be another good choice with its intrinsic advantages, simple structure and easy control. However, the influence of driving electric field to the chemiluminesence should be demonstrated prior to its utilization. Flow-rate is another factor in solution reaction. The injector must have a wide adjustable scope and good response for control instructions.

Mixing active or passive: Mixing process is vital in chemiluminesecence determination. The quick mixing leads to a drastic chemical reaction and much more photons will be emitted in short flow path and time. A high intensity will be captured by photoelectric detector in turn. In this paper the mixing length is $c a .5 \mathrm{~cm}$ because of the passive mixing, which also increases the dead volume and reagent consumption. Due to the low efficiency of passive mixing, although which is utilized in almost all the chemiluminescence chip, active mixing must be applied to microchip. But the outer powers to induce active mixing such as electric field, magnetic agitator, thermal convection, mechanical vibration and acoustic resonance must not couple with the chemiluminescence emission process. According to this, the mechanical agitator and acoustic resonance should be good candidates for active mixing chip. However, mechanical agitator is restricted in microchip due to the complicated fabrication requiements and high cost. Some primitive results of microchip mixing using surface acoustic wave (SAW) resonance are achieved and the further work is also proceeding in our research.

Temperature: Temperature is of great importance in chemical reaction. But due to the coupling of thermal stimulation and photons emission, the temperature of chemiluminescence detect is often set to room temperature in order to decrease its influence on the determination. Almost all the detections were carried out under this conditions. However, researchers also suggested that much better results could be achieved if the chemiluminescence detects were conducted at $40^{\circ} \mathrm{C}$. Following this advice, a microheator should be integrated on the chip to keep the reaction temperature constant. The balance would be erected while the thermal generated from heater must be equivalent to the loss through the surfaces of microchip. Microheaters were designed with Pt100 resistor and the test works were performed in our laboratory. The experiment results indicated that the designed microheater could warm the whole chip and make the temperature on the chip surface constant fairly with a fluctuation within $2^{\circ}$.

\section{Conclusion}

Chemiluminescence has high sensitivity and selectivity without any outer power as stimulus and has been one of the most attractive detection method in microchip based applications. In this research, microfluidic chip was fabricated with PDMS using standard MEMS techniques and flow-injection chemiluminescence experiments were performed. Sample solutions were injected into the chip with syringe and peristaltic pump, respectively. Observed results showed that the latter had a better performance in chip-based flow chemiluminescence. The conditions of experiments were optimized according to their different influences on the determination results. The standard curve of chemiluminescence intensity by benzoyl peroxide concentration was constructed from the results, which were measured from the reaction of benzoyl peroxide and luminol solutions with pre-designed concentration. Experimental results indicated that a good linear relation was formed between the chemiluminescence intensity and benzoyl peroxide concentration within $10^{-6}-10^{-8} \mathrm{~g} \mathrm{~mL}^{-1}$. The detect limit 
is about $2 \times 10^{-9} \mathrm{~g} \mathrm{~mL}^{-1}$ in benzoyl peroxide solution which was calculated according to the IUPAC. At last, flour brightener samples bought from market were tested with this microchip. The result agreed well with the benzoyl peroxide content of brightener tag, which also suggests the feasibility and accuracy of microfluidic chip-based chemiluminescence determining benzoyl peroxide application.

In contrast to the common chip, the integrated chemiluminescence chip must have the functions of sample injection, flow control, active mixing, reaction regulation, to complete the whole chemiluminescence reaction nearly automatically. Because of the coupling of the outer stimulus and the photon emission, the integrated chip was limited to some narrow ways. This project discussed some potential candidates for the microchip including inject pump, active mixer and microheater. Some potential solutions and results were also presented in this research.

\section{ACKNOWLEDGEMENTS}

This research was financially supported by the Chinese National Programs for High Technology Research and Development key project (No. 2009AA043702) and The Fundamental Research Funds for the Central Universities.

\section{REFERENCES}

1. C.Y. Liu, J. Zhang, H. Zhang and X.Q. Zhang, Appl. Chem. Ind., 38, 1064 (2009).

2. H.M. Yang, Flour Milling, 6, 41 (2007).
3. J.J. Shi and Y.F. Wang, Metrol. Measurement Technol., 10, 50 (2010).

4. C.J. Pei, G.G. Yao and W.C. Zhang, J. Anhui Agric. Sci., 24, 178 (2010).

5. H.S. Liu, W. Liu and Z.J. Zhang, J. Shaanxi Normal Univ. (Nat. Sci.), 33, 89 (2005).

6. C.J. Pei, Z.J. Zhang and W. Liu, J. Instru. Anal., 25, 83 (2006).

7. L.Q. Fang, H.F. Li and G.J. Zhang, Chin. J. Anal. Chem., 38, 1110 (2010).

8. R.F. Yan and Z.J. Zhang, Chin. J. Anal. Chem., 37, 1519 (2009).

9. Y.F. Sun, X.J. Huang, L.C. Wang Z.C. Wu and J.H. Liu, Chin. J. Spectrosc. Lab., 21, 663 (2004).

10. X.N. Han, Y.H. Tang, C.L.Yu, Y.H. Liu and H.L. Liu, J. Xi'an Jiaotong Univ. (Nat. Sci.), 26, 611 (2005).

11. T.G. Henares, F. Mizutani and H. Hisamoto, Anal. Chim. Acta, 611, 17 (2008).

12. G.M. Greenway, L.J. Nelstrop and S.N. Port, Anal. Chim. Acta, 405, 43 (2000).

13. D.Y. He, Z.J. Zhang, Y. Huang and Y.F. Hu, Food Chem., 101, 667 (2007).

14. W. Liu, Z.J. Zhang and Y. Liu, Food Chem., 95, 693 (2006).

15. A.M. Jorgensen, K.B. Mogensen, J.P. Kutter and O. Geschke, Sens. Actuators B, 90, 15 (2003).

16. X.H. Wang, M. Amatatongchai, D. Nacapricha, O. Hofmann, J.C. de Mello, D.D.C. Bradley and A.J. de Mello, Sens. Actuators B, 140, 643 (2009).

17. Y.X. Guan, Z.R. Xu, J. Dai and Z.L. Fang, Talanta, 68, 1384 (2006).

18. S.L. Zhao, Y. Huang, M. Shi, J.M. Huang and Y.M. Liu, Anal. Biochem., 393, 105 (2009).

19. X.F. Hou, Z.J. Zhang, Y. Zhao and J. Ma, J. Shaanxi Normal Univ. (Nat. Sci.), 34, 67 (2006). 